\title{
Investigating the Feasibility of Targeted Next-Generation Sequencing to Guide the Treatment of Head and Neck Squamous Cell Carcinoma
}

\section{Sun Min Lim, MD Sang Hee Cho, MD, PhD² In Gyu Hwang, $\mathrm{MD}, \mathrm{PhD}^{3}$ Jae Woo Choi, BS 4 Hyun Chang, MD, $\mathrm{PhD}^{5}$ Myung-Ju Ahn, MD, PhD Keon Uk Park, MD, $\mathrm{PhD}^{7}$ Ji-Won Kim, MD, PhD ${ }^{8}$ Yoon Ho Ko, MD, PhD ${ }^{9}$ Hee Kyung Ahn, MD ${ }^{10}$ Byoung Chul Cho, MD, PhD'1 Byung-Ho Nam, PhD12 Sang Hoon Chun, MD ${ }^{13}$ Ji Hyung Hong, MD, PhD ${ }^{14}$ Jung Hye Kwon, MD, PhD'15

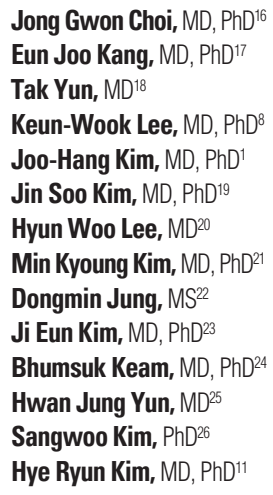
Eun Joo Kang, MD, PhD ${ }^{17}$ Tak Yun, MD ${ }^{18}$

Keun-Wook Lee, MD, PhD Joo-Hang Kim, MD, PhD ${ }^{1}$ Jin Soo Kim, MD, PhD ${ }^{19}$ Hyun Woo Lee, MD20 Min Kyoung Kim, MD, PhD21 Dongmin Jung, MS ${ }^{22}$ Ji Eun Kim, MD, $\mathrm{PhD}^{23}$ Bhumsuk Keam, MD, PhD24 Hwan Jung Yun, MD25 Sangwoo Kim, $\mathrm{PhD}^{26}$ Hye Ryun Kim, MD, PhD ${ }^{11}$

${ }^{*} A$ list author's affiliations appears at the end of the paper.

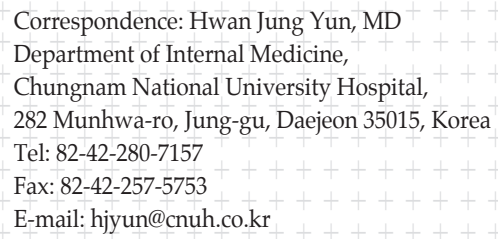

Co-correspondence: Sangwoo Kim, PhD Department of Biomedical Systems Informatics and Brain Korea 21 PLUS Project for Medical Science, Yonsei University College of Medicine,

50-1 Yonsei-ro, Seodaemun-gu, Seoul 03722, Korea Tel: 82-2-2228-0913

Fax: 82-2-2227-8129

E-mail: swkim@yuhs.ac

Co-correspondence: Hye Ryun Kim, MD, PhD Division of Medical Oncology, Department of Internal Medicine, Yonsei University College of Medicine, 50-1 Yonsei-ro, Seodaemun-gu, Seoul 03722, Korea

Tel: $82-2-2228-8125$

Fax: 82-2-393-3652

E-mail: nobelg@yuhs.ac

Received January 3, 2018

Accepted May 4, 2018

Published Online May 9, 2018

*Sun Min Lim, Sang Hee Cho, and

In Gyu Hwang contributed equally to this work.

\section{Purpose}

Head and neck squamous cell carcinoma (HNSCC) is a deadly disease in which precision medicine needs to be incorporated. We aimed to implement next-generation sequencing (NGS) in determining actionable targets to guide appropriate molecular targeted therapy in HNSCC patients.

\section{Materials and Methods}

Ninety-three tumors and matched blood samples underwent targeted sequencing of 244 genes using the Illumina HiSeq 2500 platform with an average depth of coverage of greater than 1,000×. Clinicopathological data from patients were obtained from 17 centers in Korea, and were analyzed in correlation with NGS data.

\section{Results}

Ninety-two of the 93 tumors were amenable to data analysis. TP53 was the most common mutation, occurring in 47 (51\%) patients, followed by CDKN2A ( $n=23,25 \%), \operatorname{CCND} 1(n=22$, $24 \%$ ), and PIK3CA ( $n=19,21 \%)$. The total mutational burden was similar between human papillomavirus (HPV)-negative vs. positive tumors, although TP53, CDKN2A and CCND1 gene alterations occurred more frequently in HPV-negative tumors. HPV-positive tumors were significantly associated with immune signature-related genes compared to HPV-negative tumors. Mutations of NOTCH1 ( $p=0.027), C D K N 2 A(p<0.001)$, and TP53 ( $p=0.038)$ were significantly associated with poorer overall survival. FAT1 mutations were highly enriched in cisplatin responders, and potentially targetable alterations such as PIK3CA E545K and CDKN2A R58X were noted in 14 patients (15\%).

\section{Conclusion}

We found several targetable genetic alterations, and our findings suggest that implementation of precision medicine in HNSCC is feasible. The predictive value of each targetable alteration should be assessed in a future umbrella trial using matched molecular targeted agents. 


\section{Introduction}

Head and neck squamous cell carcinoma (HNSCC) is the sixth most common malignancy worldwide, and is usually curable, if diagnosed early. Unfortunately, patients often present with advanced disease that is incurable or requires aggressive treatment, which results in functional disability, dismal prognosis and high mortality. Low survival outcomes in combination with significant toxicity of current treatment strategies emphasize the necessity for novel therapeutic modalities. Until recently, the only targeted therapy in HNSCC was cetuximab, a monoclonal antibody against epidermal growth factor receptor, which has shown a response rate of $10 \%$ to $15 \%$ in the patients with recurrent or metastatic disease [1]. However, there is no validated biomarker for predicting cetuximab efficacy, which dampens the precise selection of patients. Anti-programmed death 1 agents including pembrolizumab and nivolumab were recently approved for HNSCC that is refractory to platinum-based therapy. However, the presence of programmed death-ligand 1 (PD-L1) on tumor cells did not satisfactorily predict response, with $22 \%$ of PD-L1 positive patients responding vs. $4 \%$ of PD-L1 negative patients responding [2]. Therefore, more effective treatment strategies for personalized treatment of HNSCC are urgently needed.

Next-generation sequencing (NGS) of tumors has greatly expanded our understanding of genetic profiles, and several studies have found novel genetic alterations in HNSCC [3-6]. However, these studies were performed retrospectively in surgical specimens without incorporated clinical data on the response to therapy. Although potentially targetable genetic alterations such as PIK3CA, epidermal growth factor receptor (EGFR), and fibroblast growth factor receptor (FGFR) mutations have been identified, functional studies to validate the roles of such mutations as biomarkers remain scarce.

Herein, we describe our implementation of a precision medicine approach in 93 patients with HNSCC. This is a feasibility study of "Translational biomarker-driven umbrella project for head and neck and esophageal squamous cell carcinoma (TRIUMPH)" study by the Korean Cancer Study Group (NCT03292250) (S1 Fig.). TRIUMPH is the first, prospective, biomarker-driven umbrella trial for patients with HNSCC, consisting of multiple targeted therapies including phosphoinositide 3-kinase (PI3K) inhibitor, panHER inhibitor, FGFR inhibitor and CDK4/6 inhibitor. Patients without actionable targets are to be allocated into an immunotherapy arm. Before the start of TRIUMPH study, we conducted this feasibility study in which tumors and matched blood samples were analyzed by multiplexed targeted NGS assays. The objective of this study is to examine the feasibility of implementing NGS to guide treatment in HNSCC patients, and to find the associations between somatic alterations and clinical outcome.

\section{Materials and Methods}

\section{Patients and data collection}

Pretreatment tumor tissues (somatic) and matched normal DNA (germline) from prospectively recruited patients with HNSCC were obtained between 2016 and 2017 under the approval of Institutional Review Board of 19 institutions in Korea. HNSCC patients with initial stages 1-4 were included in this study. Clinicopathological data were collected from patient charts in accordance with an IRB-approved protocol. Clinical information including age, sex, anatomic site of tumor, tobacco and alcohol use, clinical stage, treatment history, and survival data were collected.

\section{Targeted sequencing of tumors}

Genomic DNA was isolated from formalin-fixed paraffinembedded (FFPE) samples using the QIAamp DNA FFPE Tissue Kit (Qiagen, Hilden, Germany) for the targeted sequencing of 244 head and neck cancer-related genes selected based on a literature search (S2 Table). The genomic regions of the 244 genes were captured by the customized SureSelectXT Target Enrichment library generation kit (Agilent, Santa Clara, CA), and sequenced using the Illumina HiSeq 2500 platform with a depth of coverage $>1,000 \times$.

\section{Variant calling and functional annotation}

By default, base quality trimming for short reads from the targeted sequencing was performed using Sickle [7]. Filtered reads were mapped to the human reference genome (GRCh37/hg19) using Burrows-Wheeler Aligner [8]. All reads that were mapped with $<23$ mapping quality were discarded. The aligned reads (BAM file) were further processed with the Genome Analysis ToolKit v3.5, including MarkDuplicate, Local Realignment, and Base Quality Score Recalibration [9]. Initial somatic mutations candidates were called by MuTect ver. 1.17 with a default parameter [10]. Somatic insertions/ deletions (indels) were called by Varscan2 ver. 2.3.7 with somatic $p<0.05$ [11]. From the initial call set, FoxoG artefacts were removed using the in-house Python program ver. 3.6 to discard skewed read-orientation variants [12]. The functionality of final high confidence variants was annotated with ANNOVAR software [13], including the consequences, pre- 
dicted impacts and reported allele frequencies in population. In particular, non-rare variants (minor allele frequency $>0.05$ ) were discarded to retain only pathogenic variants. Finally, the clinical interpretation of targeted therapy was annotated using the CIVIC [14] and DoCM [15] databases. Copy number alterations (CNAs) were called using CNVkit [16] for targeted deep sequencing. To reduce ambiguity from individual variations, all normal samples were pooled and used as a control. Of the initial CNA calls, genes with $\geq 4$ and 0 measured copy numbers were considered amplified and deleted, respectively, to secure high confidence. To visualize the overall landscape of mutations, 'Oncoprint' was drawn using the R package 'ComplexHeatmap' of $\mathrm{R}$ ver. 3.4. Lollipop plots were drawn for frequently mutated genes using MAFtools to check the recurrence of genomic loci with variants.

\section{Nanostring assay}

Total tumor RNA was isolated using the RNeasy kit (Qiagen). The nCounter Analysis System (Nanostring Technologies, Seattle, WA) was used to screen for the expression of 55 immune-related genes. Counts were normalized to internal controls and reference genes using the nSolver software ver. 3.0. We obtained gene expression data for 94 tumour samples, among them 8 with average expression levels of less than 10 were filtered out. The NanoStringNorm package of $\mathrm{R}$ was used for normalization [17]. We selected housekeeping.geo.mean for normalizing the samples or RNA contents. Differentially expressed genes between human papillomavirus (HPV)-positive and HPV-negative samples were identified by the glm.LRT function in the NanoStringDiff [18] package of Bioconductor. A volcano plot was drawn by using the ggplot2 package of $\mathrm{R}$. The complete list of 55 immune-related genes is shown in S3 Table.

\section{Statistical methods}

All statistical analysis was performed using the R, Python Scipy package and SPSS ver. 23.0 (IBM Corp., Armonk, NY) software. To test group-specific enrichment of genomic variants, Fisher exact test was applied to each called variant, followed by the $\mathrm{p}$-value cutoff of 0.05 . Tumor mutation burden (TMB) was measured by the number of missense mutations per megabase $(\mathrm{Mb})$ within the range of the targeted capture region. The numbers of mutations per Mb between HPV-positive and HPV-negative groups were compared using Student's t-test. Progression-free survival (PFS) and overall survival (OS) were estimated using the Kaplan-Meier method; differences between groups were compared using the logrank test. In groups of unbalanced sizes, the standard asymptotic log-rank test is often replaced by its corresponding
Table 1. Baseline characteristics of all patients

\begin{tabular}{|c|c|}
\hline Characteristic & No. $(\%)(n=93)$ \\
\hline Age, median (range, yr) & $59(28-80)$ \\
\hline \multicolumn{2}{|l|}{ Sex } \\
\hline Female & $18(19)$ \\
\hline Male & $75(81)$ \\
\hline \multicolumn{2}{|l|}{ Anatomic site } \\
\hline Oropharynx & $26(28)$ \\
\hline Oral cavity & $35(38)$ \\
\hline Hypopharynx & $15(16)$ \\
\hline Glottic larynx & $9(10)$ \\
\hline Supraglottic larynx & $3(3)$ \\
\hline Maxillary sinus & $5(5)$ \\
\hline \multicolumn{2}{|l|}{ Tobacco use } \\
\hline Never & $26(28)$ \\
\hline Former & $49(53)$ \\
\hline Current & $18(19)$ \\
\hline \multicolumn{2}{|l|}{ Alcohol use } \\
\hline Never & $34(37)$ \\
\hline Former & $33(35)$ \\
\hline Current & $26(28)$ \\
\hline \multicolumn{2}{|c|}{ Clinical stage at initial diagnosis } \\
\hline I-III & $54(58)$ \\
\hline IV & $39(42)$ \\
\hline \multicolumn{2}{|l|}{ HPV status } \\
\hline Positive & $20(22)$ \\
\hline Negative & $56(60)$ \\
\hline Unknown & $17(18)$ \\
\hline Prior surgery & $68(73)$ \\
\hline
\end{tabular}

HPV, human papillomavirus.

permutation test; alternatively, the distribution under the null hypothesis is approximated via Monte Carlo resampling. Here, we used empirical p-values from 10,000 replicates by using the log-rank test function in the coin package of $R$. Two-sided $p$-values of $<0.05$ were considered significant.

\section{Ethical statement}

This study was conducted under the approval of Institutional Review Board of 19 institutions in Korea. All patients provided written informed consent for genomic testing used for this study. 


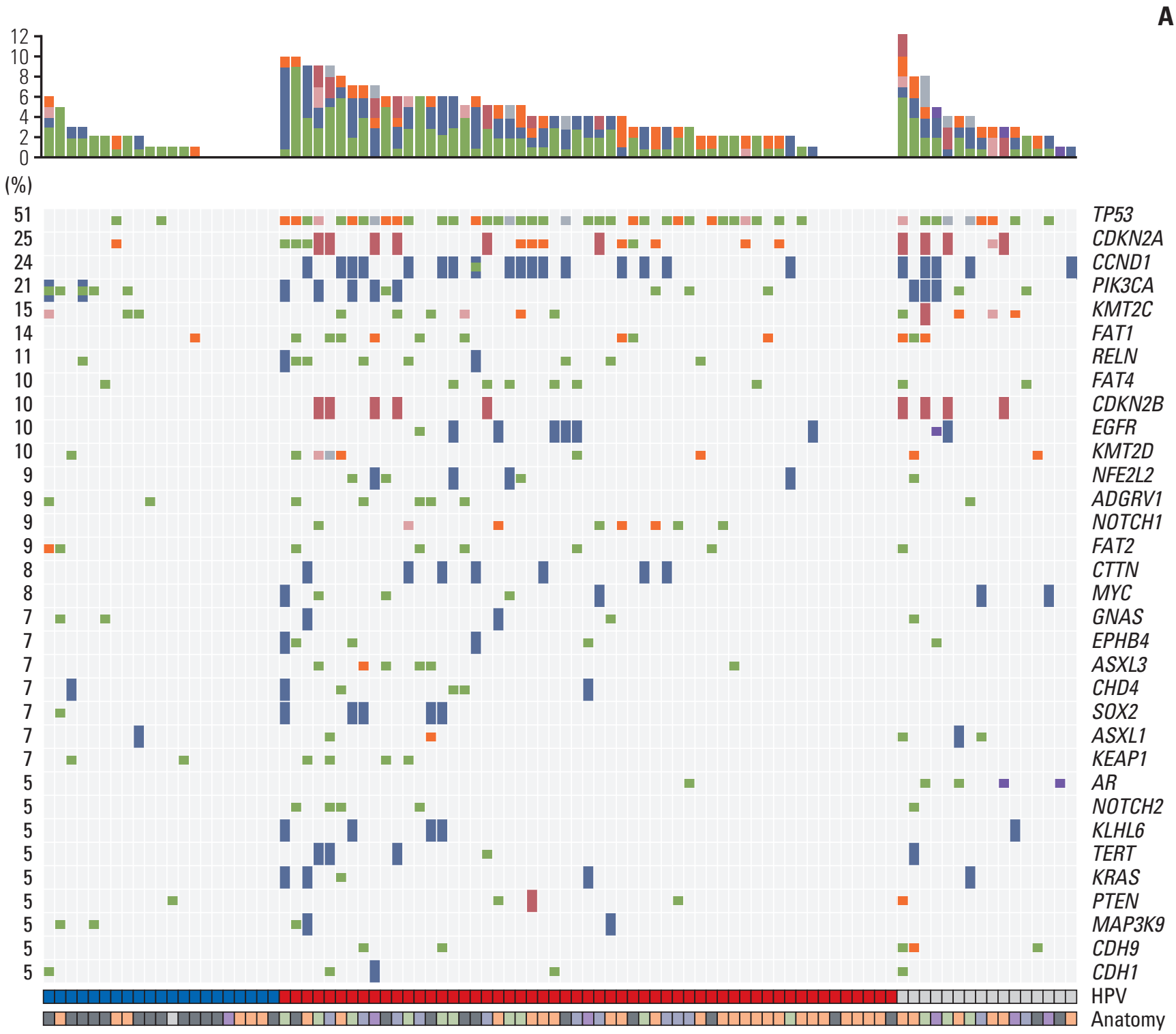

Alterations $\square$ Amplification $\square$ Deletion $\square$ Missense $\square$ Nonsense $\square$ Frame shift indel $\square$ In-frame indel $\square$ Splice site

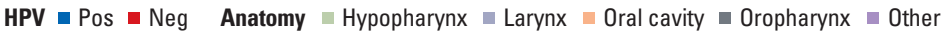

Fig. 1. (A) Mutational spectrum and copy number alterations in head and neck squamous cell carcinomas detected by targeted sequencing. Samples with a greater than $1 \%$ incidence of genetic alterations are shown, and are stratified by human papillomavirus (HPV) status and primary tumor anatomic site. Pos, positive; Neg, negative. (Continued to the next page)

\section{Results}

\section{Clinical characteristics}

A total of 93 patient tumors were included in 75 men and 18 women. Clinical data are summarized in Table 1; the median age of all patients was 59 years (range, 28 to 80 years) 


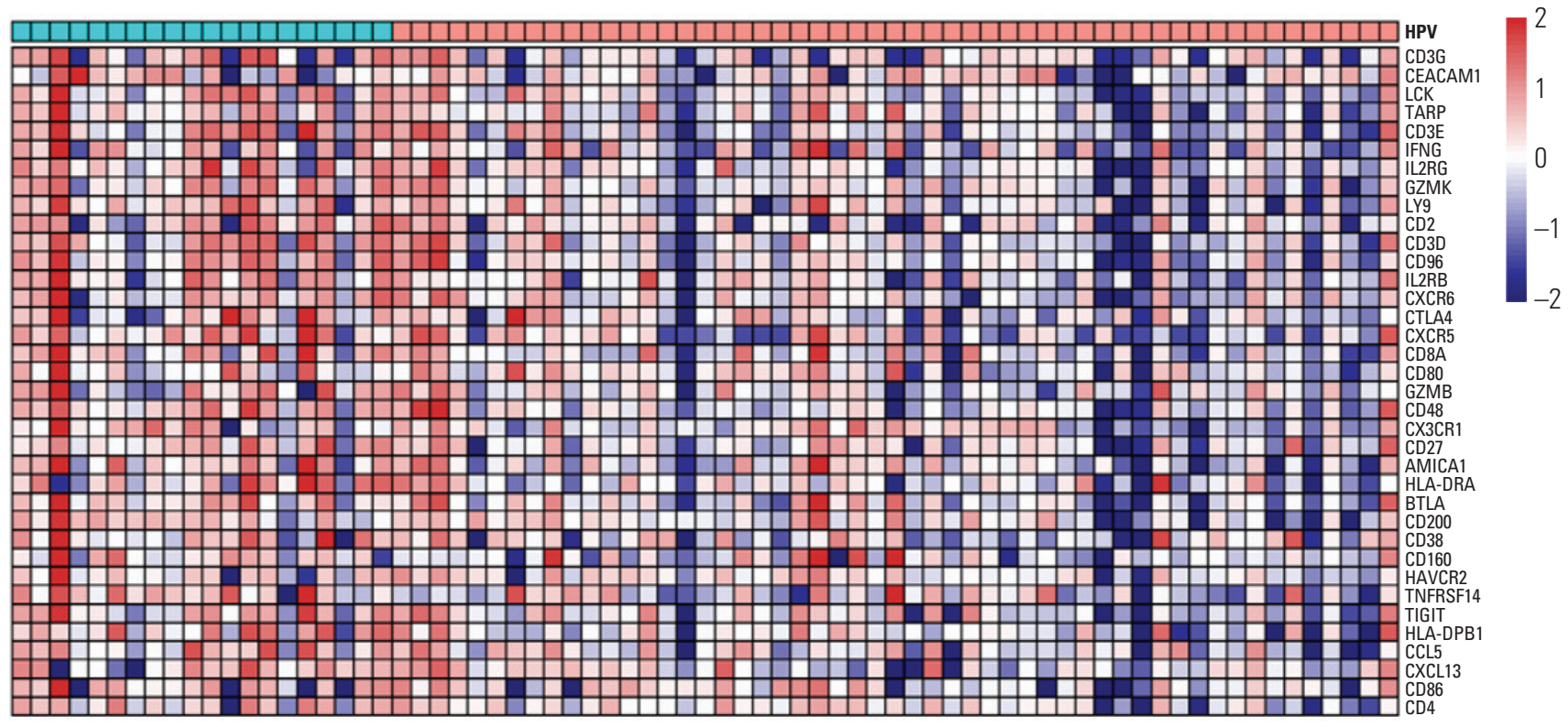

C

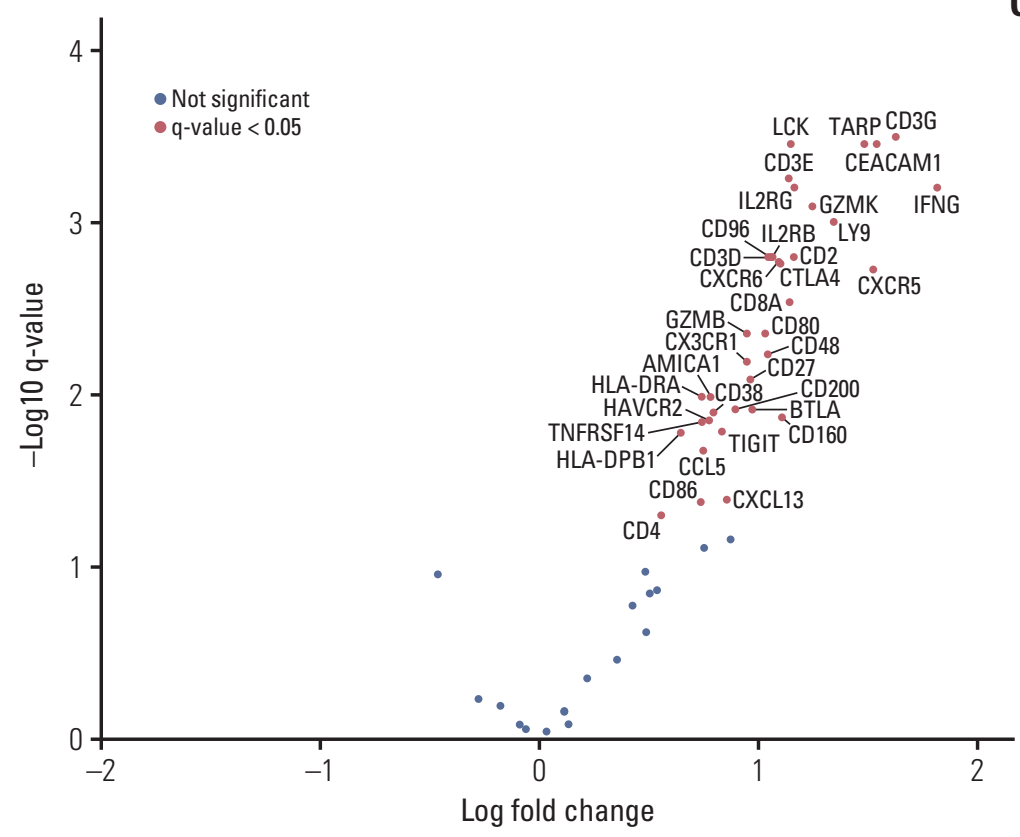

Fig. 1. (Continued from the previous page) (B) A heat map of 55 differentially expressed genes with an absolute fold change $\geq 2$ and a false discovery rate $(F D R)<0.05$. (C) Volcano plot showing the distribution of the fold changes in gene expression. Genes with an absolute fold change $\geq 2$ and FDR $<0.05$ are indicated in red (high expression in HPV-positive tumors compared to HPV-negative tumors). 

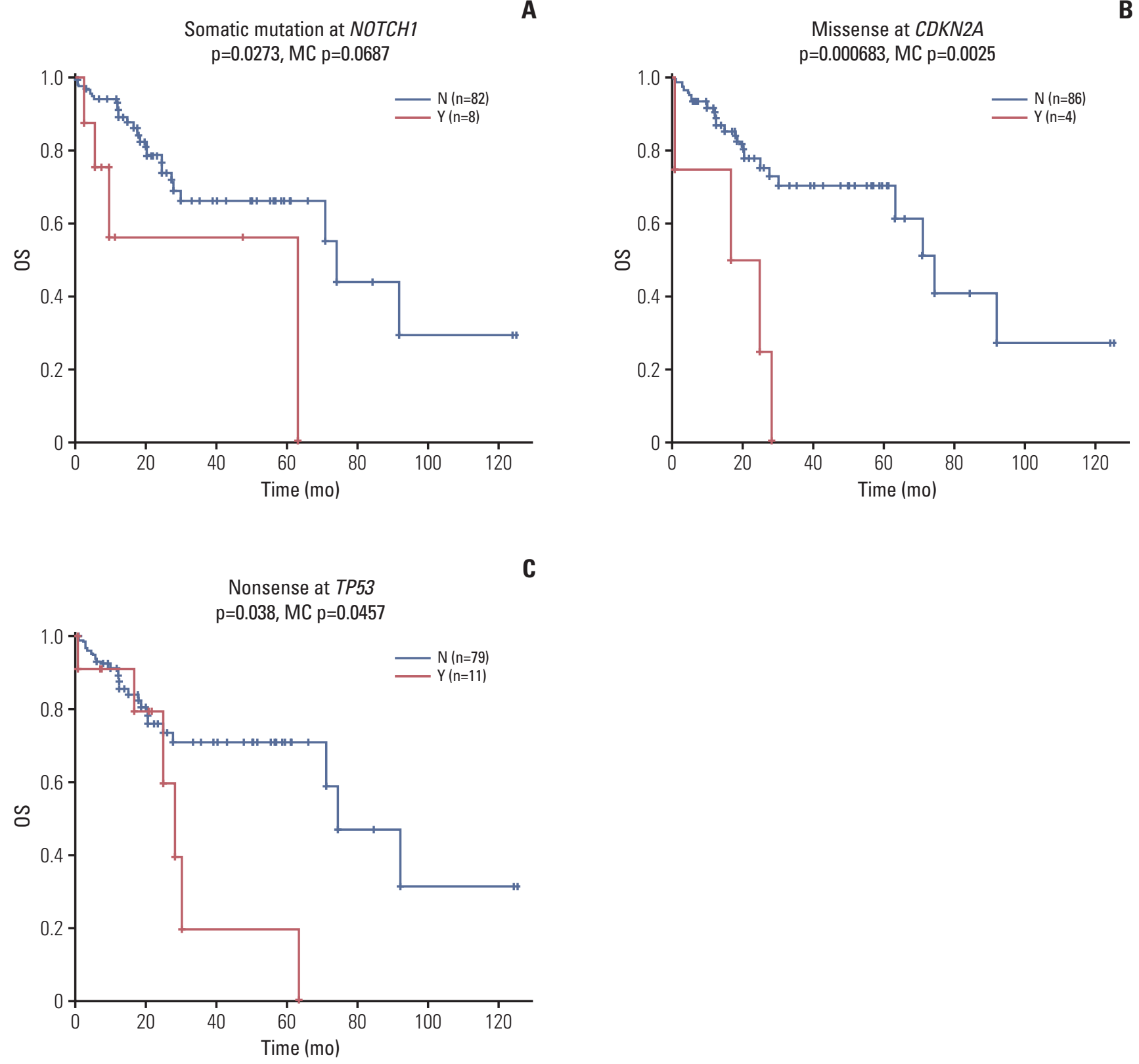

Fig. 2. Kaplan-Meier curves showing the association of single nucleotide variations and overall survival (OS) in patients. (A) Patients with NOTCH1 somatic mutation had poorer overall survival (somatic mutation includes missense, nonsense, splice site mutations, frame shift indels, or in-frame indels). (B) Patients with CDKN2A missense mutations had poorer OS. (C) Patients with TP53 nonsense mutation showed poorer OS.

performed for locoregional recurrence, and systemic chemotherapy was performed for metastatic disease. For the whole cohort, the median PFS and OS were 12.5 months (95\% confidence interval [CI], 10.2 to 14.8$)$ and 70 months $(95 \% \mathrm{CI}$, 57.4 to 84.4 ), respectively, with a median follow-up of 20 months. Patients with HPV-positive oropharynx cancers $(n=16)$ showed a better 2-year OS rate than HPV-negative patients ( $n=10)(31 \%$ vs. $10 \%$, respectively), although the difference was not significant owing to the small number of cases. 


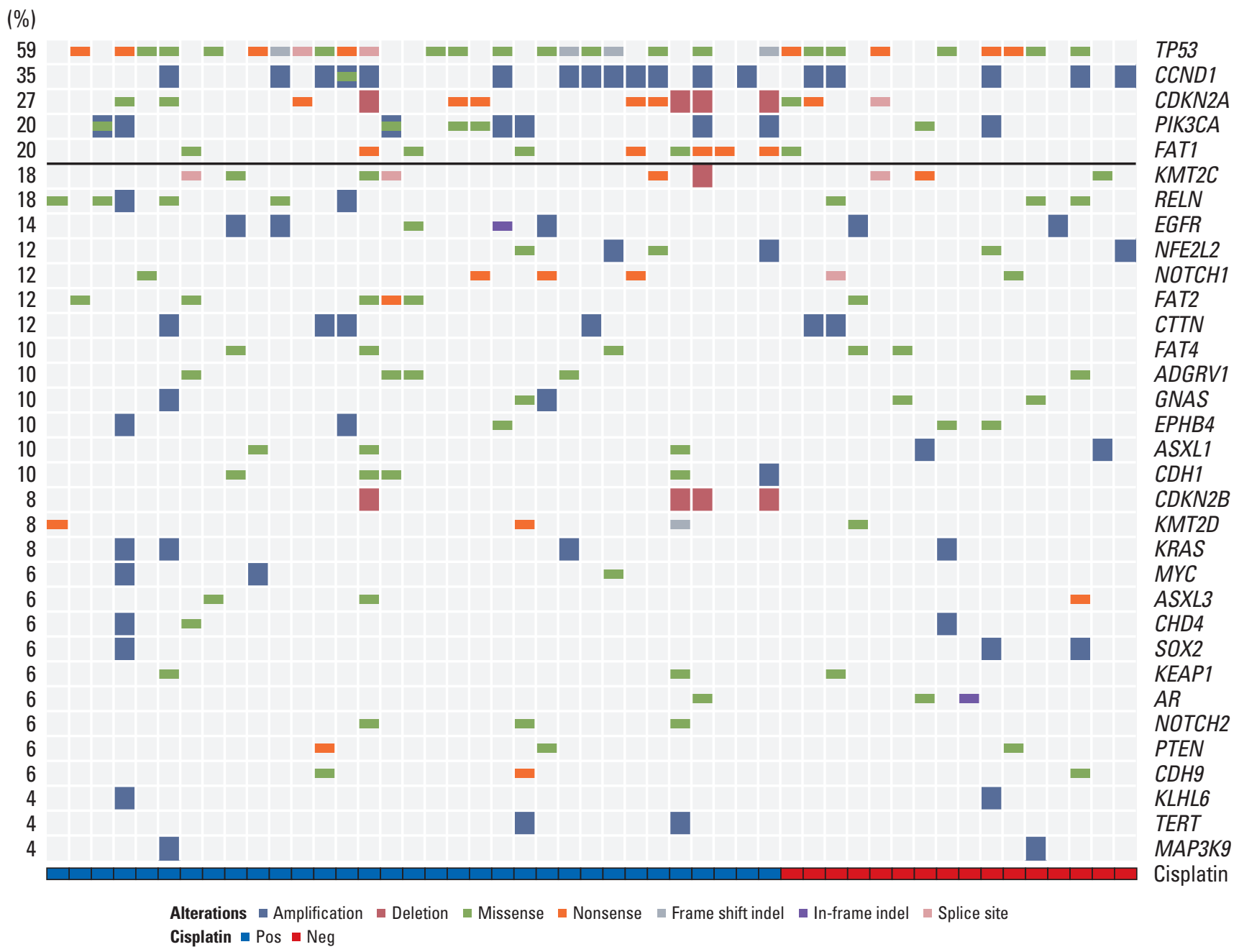

Fig. 3. Patients who received cisplatin-based chemotherapy were categorized into responders vs. non-responders and genetic alterations are shown in the order of frequency. Pos, positive; Neg, negative.

\section{Overview of somatic mutations in HNSCC}

A total of 2,315 somatic single nucleotide variations (SNVs) and 19 indels were identified from the targeted sequencing of the 92 tumors, which corresponds to a rate of 3.64 SNVs per $1 \mathrm{Mb}$. We found that TP53 was the most frequently mutated gene $(\mathrm{n}=47,51 \%)$, followed by CDKN2A $(\mathrm{n}=23$, $25 \%), C C N D 1(\mathrm{n}=22,24 \%)$, and PIK3CA $(\mathrm{n}=19,21 \%)$ (Fig. 1A). As expected, smokers displayed a significantly higher TMB than non-smokers (4.16/Mb vs. 3.12/Mb, p=0.04) (S4 Fig.).

\section{Comparison of HPV-positive vs. HPV-negative tumours}

Of 92 patient tumors, 76 tumors ( $82 \%$ ) had known HPV status and we compared molecular landscape of HPV-positive and HPV-negative tumors. TMB counts were higher in
HPV-negative than HPV-positive tumors, although the difference was not significant (4.16/Mb vs. 3.12/Mb, $\mathrm{p}=0.150)$ (S5 Fig.). TP53, CDKN2A, and CCND1 gene alterations were significantly more frequent in HPV-negative tumors (Fig. 1A). As described previously, we observed TP53 mutations among HPV-negative tumors at higher rates than HPV-positive tumors $(65.5 \%$ vs. $9.5 \%, \mathrm{p}<0.001)$. Inactivating mutations such as $C D K N 2 A$ and $C D K N 2 B$ deletions $(\mathrm{n}=6)$, and $C C N D 1$ amplification ( $\mathrm{n}=17$ ) were exclusively identified in HPV-negative tumors. We also noted HPV-negative specific genetic alterations in receptor tyrosine kinases (RTKs) including EGFR, FGFR1/3, and platelet-derived growth factor receptor A (PDGFRA), which was consistent with a previous study [5]. PIK3CA mutations were more commonly found in HPVpositive tumors $(23.8 \%$ vs. $16.4 \%, \mathrm{p}>0.05)$. Comparison of immune signatures between HPV-positive and HPV-nega- 


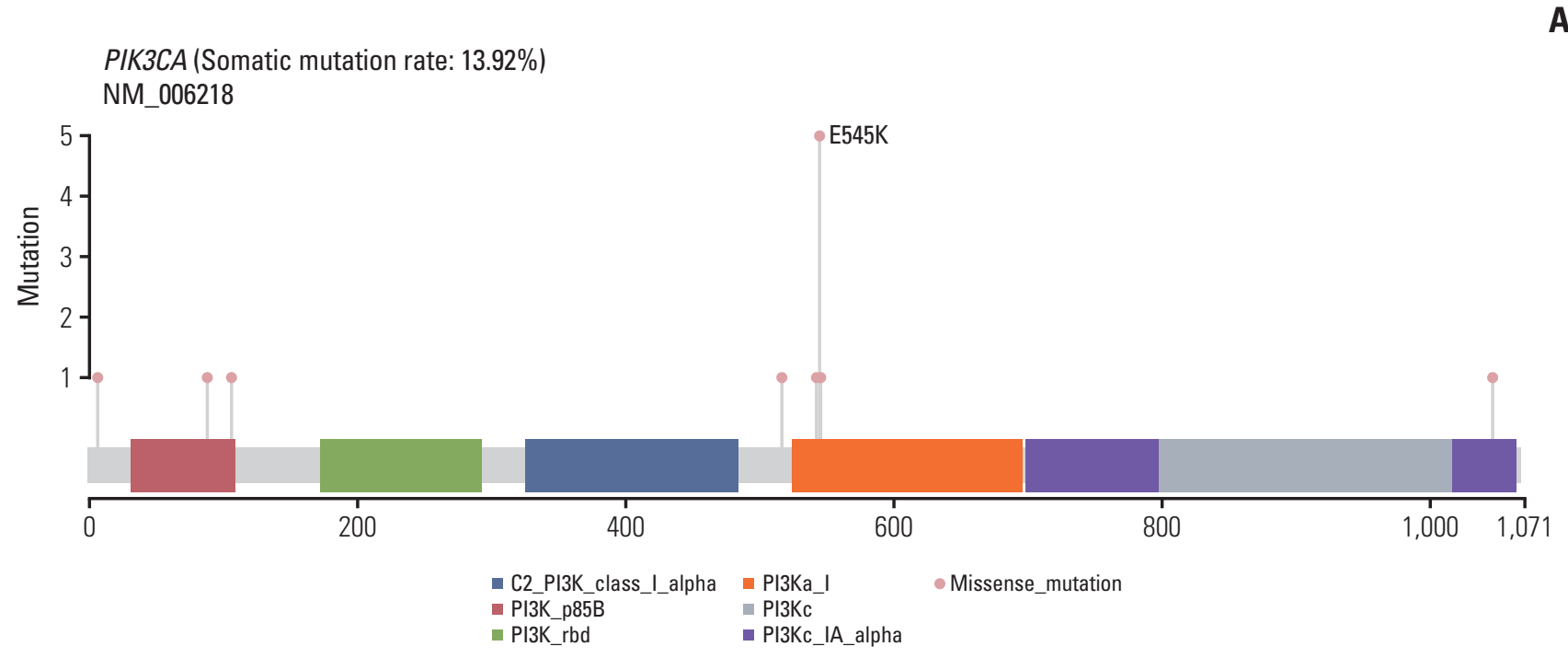

CDKN2A (Somatic mutation rate: $16.46 \%$ ) NM_000077

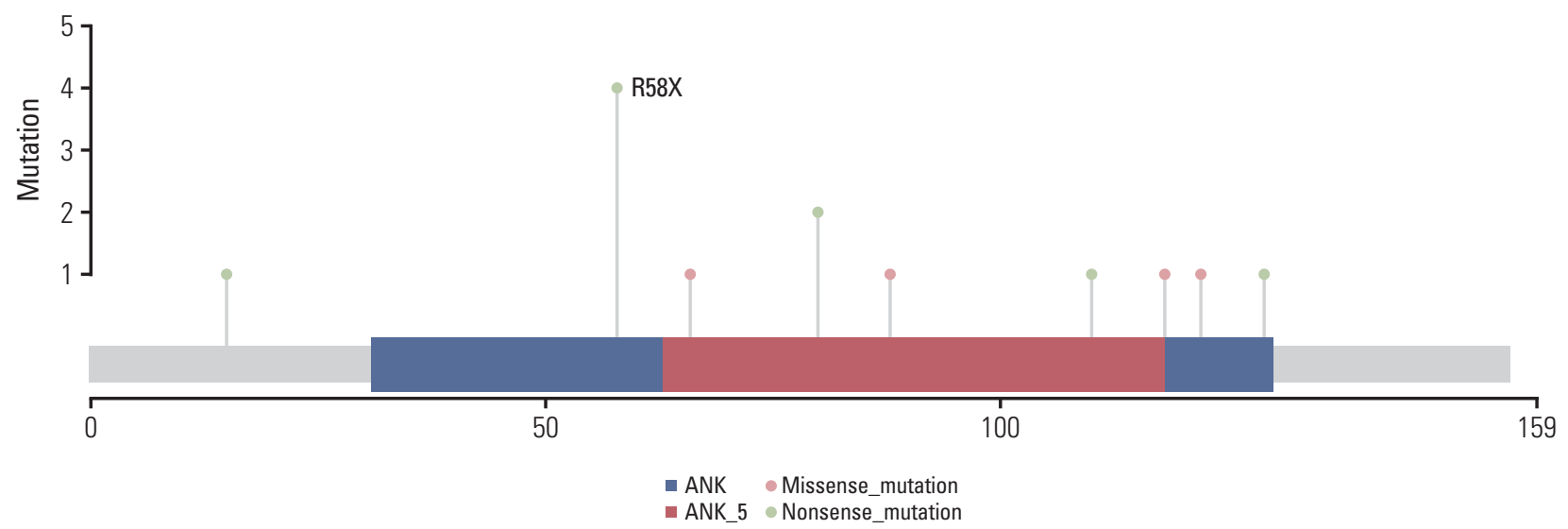

Fig. 4. Gene diagrams for a selection of key mutations in potentially targetable genes PIK3CA (A), CDKN2A (B), and TP53 (C). (D) Signaling pathway deregulation is shown. HPV, human papillomavirus. (Continued to the next page)

tive tumors via nanostring assay revealed that HPV-positive tumors were significantly enriched with immune-related genes. HPV-tumors harbored higher levels of immune activation: specifically, CD3 $\left(\mathrm{p}=6.0 \times 10^{-6}\right), \operatorname{CECAM} 1\left(\mathrm{p}=4.9 \times 10^{-5}\right)$ and IL2R $\left(p=6.9 \times 10^{-5}\right)$ expression (Fig. 1B and C).

\section{Clinical correlation}

We performed an exploratory analysis to correlate gene alterations (SNVs and CNAs) with survival (Fig. 2). In 90 patients with available survival data, genomic events associated with poorer OS were mutations in NOTCH1 $(\mathrm{p}=0.027)$,
CDKN2A $(\mathrm{p}<0.001)$, and TP53 $(\mathrm{p}=0.038)$. The association between CDKN2A, TP53 mutations and poor OS was consistent with a previous analysis of The Cancer Genome Atlas (TCGA) database. CNAs were not associated with any gene alterations. In contrast to a previous report [19], PIK3CA amplification was not associated with worse OS (S6 Fig.).

Next, we analyzed gene alterations associated with cisplatin resistance by classifying patients who received cisplatin-based chemotherapy into responders and non-responders. According to Response Evaluation Criteria in Solid Tumors (ver. 1.1), responders were patients who showed complete response, partial response or stable disease to cis- 
P53 (Somatic mutation rate: $59.49 \%$ )

NM_000546

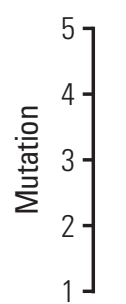

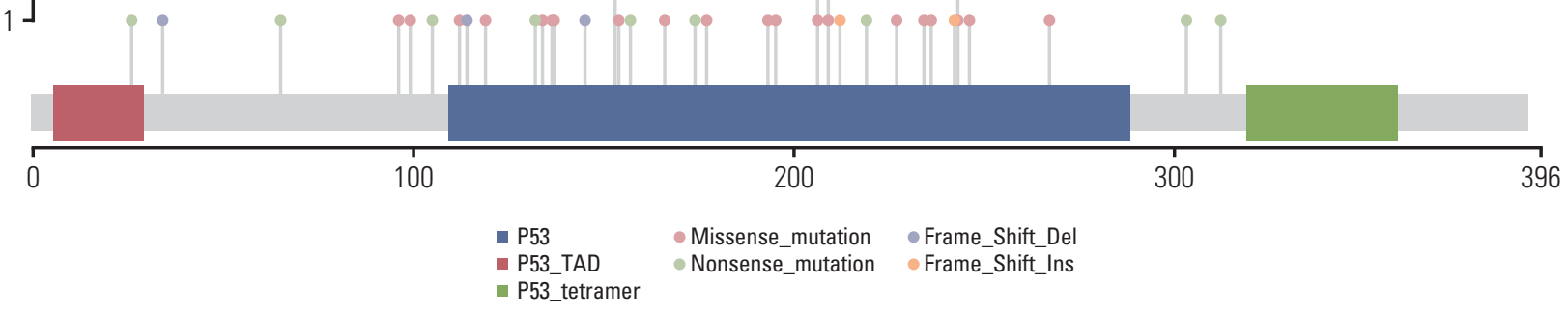

D

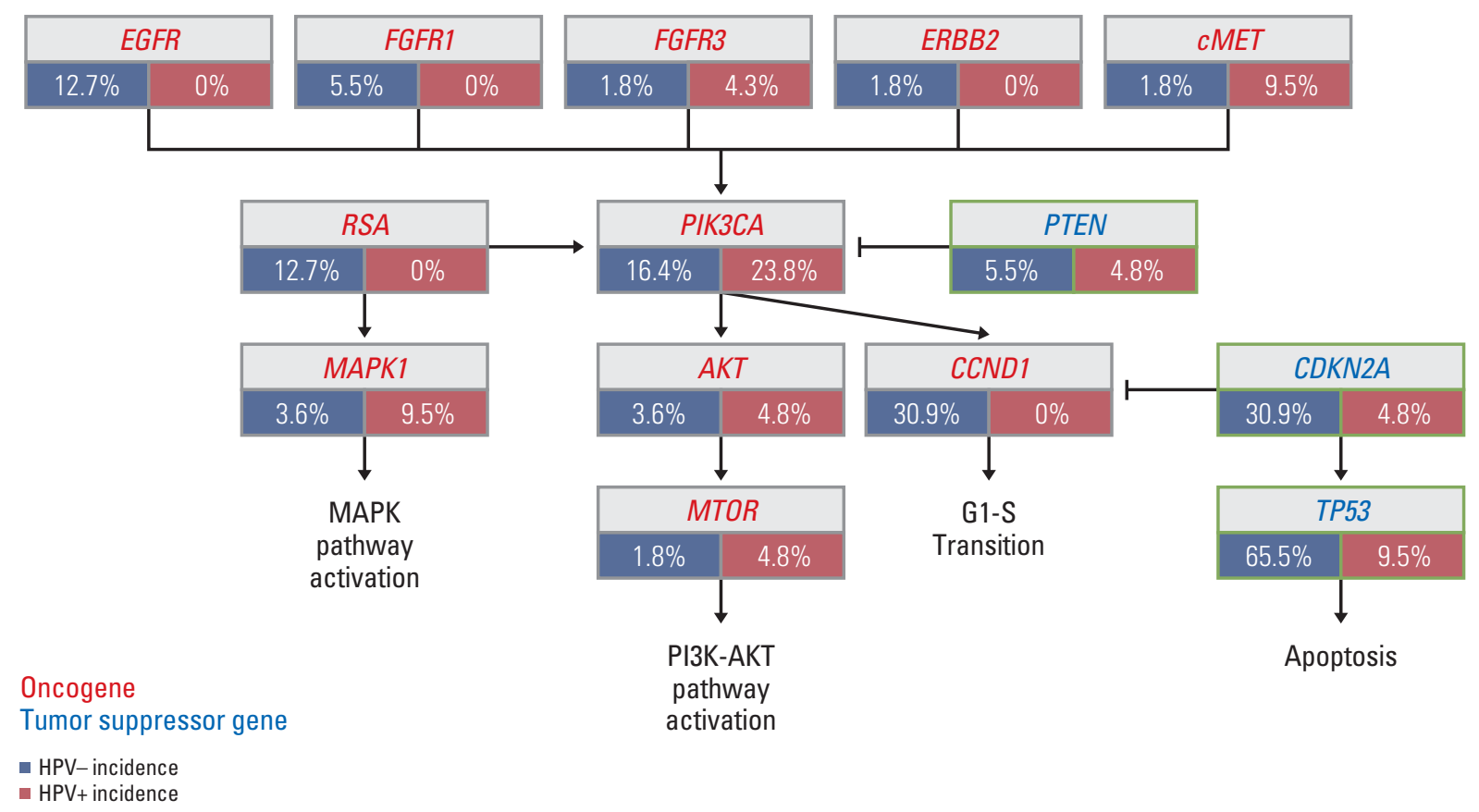

Fig. 4. (Continued from the previous page)

platin-based chemotherapy, whereas non-responders were those with progressive disease [20]. Among 54 evaluable patients, $38(70 \%)$ were responders, and $16(30 \%)$ were nonresponders. FAT1 gene mutations (5 missense and 5 nonsense) were highly enriched in cisplatin responders compared to non-responders $(\mathrm{p}<0.05)$ (Fig. 3, S7 Fig.).

\section{Targetable mutations and copy-number aberrations}

We identified potentially targetable mutations in PIK3CA and CDKN2A. An established canonical mutation, PIK3CA E545K missense mutation were identified in five patients (5\%) (Fig. 4A), while CDKN2A R58X nonsense mutation was identified in four patients (4\%) (Fig. 4B). TP53 inactivating 
mutations (R209Q/W, R243W/Q) that cause cell cycle deregulation occurred in eight patients (5\%) (Fig. 4C).

Fig. 4D summarizes the deregulated signaling pathways. Among RTKs, EGFR and $c M E T$ alterations were frequent, followed by FGFR3, FGFR1, and ERBB2. Among downstream targets of the RTKs/RAS/PI3K pathway, PIK3CA dominated with occasional MAPK1 and MTOR mutations. RAS and MAPK1 alterations occurred in $12.7 \%$ and $13.1 \%$ of patients, respectively. Alterations in tumor suppressors, TP53 and CDKN2A were notable in HPV-negative tumors, which were consistent with a recent report [21]. Overall, alterations in genes involved in cell death and PIK3CA/AKT/ MTOR pathway were predominant.

\section{Discussion}

Our umbrella trial suggests that using NGS for determining treatment strategies for patients with HNSCC is feasible, and that translating genomic data into clinical care is attainable. The most common genomic alterations (TP53, PIK3CA, $C C N D 1$, and $C D K N 2 A$ ) were identified at frequencies consistent with investigations of TCGA. Previous studies have characterized mostly surgically resected HNSCC samples, with a limited portion of HPV-positive samples. TCGA study, which is the largest cohort to date $(n=279)$ is comprised of surgically resected oral cavity or laryngeal squamous cell carcinoma patients, and treatment and survival data were limited [5]. Recently, Seiwert et al. [22] reported a large number of HPV-positive tumors, where they included $51(42.5 \%) \mathrm{HPV}$-positive patients in a total of 120 patients. Consistent with our finding, the mutational burdens in HPVpositive and -negative tumors were similar, while FGFR2 aberrations were exclusively identified in HPV-negative tumors.

Our study emphasizes how the application of NGS may be used as a prospective, master protocol tailored to each patient's genotype. The turnaround time from patient sample collection to NGS results was within 4 weeks, which is timely for patient enrolment. Similarly, another study recently found it feasible to incorporate NGS into the clinical care of HNSCC patients [19]. Patients who received targeted therapy matched to their genotypes achieved a higher objective response rate than patients unmatched to therapy. However, they used two different NGS platforms with inconsistent mutation rates and actionable alterations. Additionally, the MOSCATO-01 trial showed that genomic analyses of 199 patients with advanced cancers produced improved outcomes with matched targeted therapy [23]. The ongoing NCI-MATCH trial is currently assessing whether molecular markers can predict response to targeted therapies in patients with advanced cancer [24] and the results are awaited.

PI3K pathway aberrations are potential therapeutic targets in HNSCC patients. Prior studies identified that PIK3CA mutation or amplification was associated with various clinical outcomes. One study reported that PIK3CA amplification was associated with significantly decreased PFS, whereas PIK3CA mutation was not [19]. Another study demonstrated that PIK3CA mutations were correlated with poor prognosis in HPV-negative, locally advanced HNSCC [22]. A preclinical study also reported that patient-derived PIK3CA mutant HNSCC tumor grafts are potentially sensitive to PI3K/mTOR inhibitors [25]. In our cohort, patients with a PIK3CA hotspot mutation (E545K) will be treated with the PI3K pathway inhibitor (BYL719).

Deletion of CDKN2A or amplification of CCND1, which induces sustained CDK $4 / 6$ activation, occurred at $27 \%$ and $22 \%$, respectively, which were comparable to such cell-cycle related gene aberrations found in other studies [5,22]. Preclinical or clinical data regarding the activity of CDK inhibitor in HNSCC is limited, but our prospective trial may solve which genotypes will benefit from treatment with CDK inhibitors.

FAT atypical cadherin 1 (FAT1) was significantly enriched in cisplatin responders. FAT1 gene has been reported to be associated with various types of cancer, including HNSCC $[5,26]$. FAT1 gene acts as a tumor suppressor, in which lossof-function activates Wnt pathway and tumorigenesis [27]. Recently, FAT1 mutation was significantly associated with better OS in HPV-negative patients from both the TCGA cohort and the International Cancer Genome Consortium (ICGC) data cohort [28]. The functional impact of the FAT1 mutation identified in our study requires further investigation to determine its role as a prognostic or predictive biomarker.

In our study, immune signatures were highly enriched in HPV-positive tumors, consistent with a previous finding that HPV-positive tumors have a distinct immune phenotype, characterized by more immune cell infiltration and higher levels of $\mathrm{CD}^{+}$T-cell activation [29]. As ongoing checkpoint inhibitor trials (NCT02105636, NCT01848834) showed promising preliminary activities in HNSCC patients, improved outcome in HPV-positive patients may be related to their immunophenotype [30,31].

The accuracy and fidelity of genomic analysis are critical; therefore, false-positive or false-negative genomic variants should be carefully avoided. To that end, several technical issues were noted in our study. First, the often inevitable low tumour cellularity in samples, owing to normal cell contamination, has a negative effect on the accuracy of calling of SNVs and CNVs [32]. We found that, among 92 samples, 14 
$(15 \%)$ and five $(5 \%)$ obtained via core needle biopsy and excision, respectively, showed low tumor cellularity $(\sim 30 \%)$. As CNV analysis is directly affected by reduced cellularity, CNVs with ambiguous analysis scores may require confirmation using alternative methods. Second, sequencing artefacts can appear in every step of the NGS pipeline, which complicates the differentiation between true vs. false variants. We observed an abnormally excessive number of lowlevel somatic mutations in a few samples (mutation-rate $/ \mathrm{Mb}$ $>100$ ), which could only be removed using an oxoG filtering program [12]. Such false variants can distort the overall distribution of somatic mutations and their relative burdens, and should be specially inspected via advanced bioinformatics analyses. Third, whole-exome or targeted sequencing for identifying CNVs remain secondary options, as more sensitive methods such as whole-genome sequencing or specialized array-based methods are widely unavailable. As targeted sequencing based CNV analysis generally performs better in a larger cohort, size and sustainability of clinical trials should be considered when they are designed. Moreover, active participation of genome analysis experts is highly recommended to manage such technical issues.

In conclusion, our large-scale targeted sequencing of HNSCC patient samples identified potentially targetable alterations. Further prospective validation of NGS based molecularly targeted treatment is highly warranted.

\section{Electronic Supplementary Material}

Supplementary materials are available at Cancer Research and Treatment website (https:// www.e-crt.org).

\section{Conflicts of Interest}

Conflict of interest relevant to this article was not reported.

\section{Acknowledgments}

This study was supported by a grant from the National R\&D Program for Cancer Control, Ministry of Health and Welfare, Republic of Korea (1631050). The funder had no role in the study design; in the collection, analysis and interpretation of data; in the writing of the report; or in the decision to submit the article for publication.

\section{Author Details}

${ }^{1}$ Division of Medical Oncology, Department of Internal Medicine, CHA Bundang Medical Center, Seongnam, ${ }^{2}$ Department of HematoOncology, Chonnam National University Hwasun Hospital, Hwasun, ${ }^{3}$ Division of Hematology / Oncology, Department of Internal Medicine, Chung-Ang University Hospital, Chung-Ang University College of Medicine, Seoul, ${ }^{4}$ Department of Pharmacology, Sever- ance Biomedical Science Institute, Yonsei University of College of Medicine, Yonsei Cancer Research Institute, JE-UK Laboratory of Molecular Cancer Therapeutics, Seoul, ${ }^{5}$ Division of Hematology and Medical Oncology, International St. Mary's Hospital, Catholic Kwandong University College of Medicine, Incheon, ${ }^{6}$ Department of Medicine, Samsung Medical Center, Sungkyunkwan University School of Medicine, Seoul, 'Department of Hemato-Oncology, Keimyung University Dongsan Medical Center, Daegu, ${ }^{8}$ Department of Internal Medicine, Seoul National University Bundang Hospital, Seongnam, ${ }^{9}$ Department of Internal Medicine, Uijeongbu St. Mary's Hospital, Uijeongbu, ${ }^{10}$ Department of Medical Oncology, Gachon University Gil Medical Center, Incheon, ${ }^{11}$ Divison of Medical Oncology, Department of Internal Medicine, Yonsei Cancer Center, Yonsei University College of Medicine, Seoul, ${ }^{12}$ HERINGS, The Institute of Advanced Clinical \& Biomedical Research, Seoul, ${ }^{13}$ Division of Medical Oncology, Department of Internal Medicine, Bucheon St. Mary's Hospital, College of Medicine, The Catholic University of Korea, Bucheon, ${ }^{14}$ Department of Internal Medicine, Incheon St. Mary's Hospital, Incheon, ${ }^{15}$ Division of Hemato-Oncology, Department of Internal Medicine, Hallym University Kangdong Sacred Heart Hospital, Hallym University College of Medicine, Seoul, ${ }^{16}$ Department of Internal Medicine, Konyang University Hospital, Daejeon, ${ }^{17}$ Division of Medical Oncology, Department of Internal Medicine, Korea University Guro Hospital, Korea University College of Medicine, Seoul, ${ }^{18}$ Rare Cancers Clinic, Center for Specific Organs Cancer, National Cancer Center, Goyang, ${ }^{19}$ Department of Internal Medicine, SMG-SNU Boramae Hospital, Seoul, ${ }^{20}$ Department of Hematology-Oncology, Ajou University School of Medicine, Suwon, ${ }^{21}$ Department of Internal Medicine, Yeungnam University College of Medicine, Daegu, ${ }^{22}$ Department of Pharmacology, Institute for Cancer Research, Yonsei Cancer Center, Yonsei University College of Medicine, Seoul, ${ }^{23}$ Department of Pathology, SMG-SNU Boramae Hospital, Seoul National University College of Medicine, Seoul, ${ }^{24}$ Department of Internal Medicine, Seoul National University Hospital, Seoul, ${ }^{25}$ Department of Internal Medicine, Chungnam National University Hospital, Daejeon, ${ }^{26}$ Department of Biomedical Systems Informatics and Brain Korea 21 PLUS Project for Medical Science, Yonsei University College of Medicine, Seoul, Korea 


\section{References}

1. Vermorken JB, Trigo J, Hitt R, Koralewski P, Diaz-Rubio E, Rolland F, et al. Open-label, uncontrolled, multicenter phase II study to evaluate the efficacy and toxicity of cetuximab as a single agent in patients with recurrent and/or metastatic squamous cell carcinoma of the head and neck who failed to respond to platinum-based therapy. J Clin Oncol. 2007;25: 2171-7.

2. Chow LQ, Haddad R, Gupta S, Mahipal A, Mehra R, Tahara $\mathrm{M}$, et al. Antitumor activity of pembrolizumab in biomarkerunselected patients with recurrent and/or metastatic head and neck squamous cell carcinoma: results from the phase Ib KEYNOTE-012 Expansion Cohort. J Clin Oncol. 2016;34:3838-45.

3. Agrawal N, Frederick MJ, Pickering CR, Bettegowda C, Chang $\mathrm{K}$, Li RJ, et al. Exome sequencing of head and neck squamous cell carcinoma reveals inactivating mutations in NOTCH1. Science. 2011;333:1154-7.

4. Stransky N, Egloff AM, Tward AD, Kostic AD, Cibulskis K, Sivachenko A, et al. The mutational landscape of head and neck squamous cell carcinoma. Science. 2011;333:1157-60.

5. Cancer Genome Atlas Network. Comprehensive genomic characterization of head and neck squamous cell carcinomas. Nature. 2015;517:576-82.

6. Giefing M, Wierzbicka M, Szyfter K, Brenner JC, Braakhuis BJ, Brakenhoff $\mathrm{RH}$, et al. Moving towards personalised therapy in head and neck squamous cell carcinoma through analysis of next generation sequencing data. Eur J Cancer. 2016;55:14757.

7. Joshi NA, Fass JN. Sickle: a sliding-window, adaptive, quality-based trimming tool for FastQ files (version 1.33) [Internet]. San Francisco, CA: GitHub Inc.; 2011 [cited 2018 Jan 1]. Available from: https://github.com/najoshi/sickle.

8. Li H, Durbin R. Fast and accurate short read alignment with Burrows-Wheeler transform. Bioinformatics. 2009;25:1754-60.

9. McKenna A, Hanna M, Banks E, Sivachenko A, Cibulskis K, Kernytsky A, et al. The Genome Analysis Toolkit: a MapReduce framework for analyzing next-generation DNA sequencing data. Genome Res. 2010;20:1297-303.

10. Cibulskis K, Lawrence MS, Carter SL, Sivachenko A, Jaffe D, Sougnez C, et al. Sensitive detection of somatic point mutations in impure and heterogeneous cancer samples. Nat Biotechnol. 2013;31:213-9.

11. Koboldt DC, Zhang Q, Larson DE, Shen D, McLellan MD, Lin L, et al. VarScan 2: somatic mutation and copy number alteration discovery in cancer by exome sequencing. Genome Res. 2012;22:568-76.

12. Costello M, Pugh TJ, Fennell TJ, Stewart C, Lichtenstein L, Meldrim JC, et al. Discovery and characterization of artifactual mutations in deep coverage targeted capture sequencing data due to oxidative DNA damage during sample preparation. Nucleic Acids Res. 2013;41:e67.

13. Wang K, Li M, Hakonarson H. ANNOVAR: functional annotation of genetic variants from high-throughput sequencing data. Nucleic Acids Res. 2010;38:e164.

14. Griffith M, Spies NC, Krysiak K, McMichael JF, Coffman AC,
Danos AM, et al. CIViC is a community knowledgebase for expert crowdsourcing the clinical interpretation of variants in cancer. Nat Genet. 2017;49:170-4.

15. Ainscough BJ, Griffith M, Coffman AC, Wagner AH, Kunisaki J, Choudhary MN, et al. DoCM: a database of curated mutations in cancer. Nat Methods. 2016;13:806-7.

16. Talevich E, Shain AH, Botton T, Bastian BC. CNVkit: genomewide copy number detection and visualization from targeted DNA sequencing. PLoS Comput Biol. 2016;12:e1004873.

17. Waggott DM. NanoString Norm: normalize NanoString miRNA and mRNA data. R Package; 2015.

18. Wang H, Zhai T, Wang C, NanoStringDiff: differential expression analysis of NanoString nCounter data. R Package; 2015.

19. Chau NG, Li YY, Jo VY, Rabinowits G, Lorch JH, Tishler RB, et al. Incorporation of next-generation sequencing into routine clinical care to direct treatment of head and neck squamous cell carcinoma. Clin Cancer Res. 2016;22:2939-49.

20. Eisenhauer EA, Therasse P, Bogaerts J, Schwartz LH, Sargent D, Ford R, et al. New response evaluation criteria in solid tumours: revised RECIST guideline (version 1.1). Eur J Cancer. 2009;45:228-47.

21. Chung CH, Guthrie VB, Masica DL, Tokheim C, Kang H, Richmon J, et al. Genomic alterations in head and neck squamous cell carcinoma determined by cancer gene-targeted sequencing. Ann Oncol. 2015;26:1216-23.

22. Seiwert TY, Zuo Z, Keck MK, Khattri A, Pedamallu CS, Stricker $\mathrm{T}$, et al. Integrative and comparative genomic analysis of HPV-positive and HPV-negative head and neck squamous cell carcinomas. Clin Cancer Res. 2015;21:632-41.

23. Massard C, Michiels S, Ferte C, Le Deley MC, Lacroix L, Hollebecque $\mathrm{A}$, et al. High-throughput genomics and clinical outcome in hard-to-treat advanced cancers: results of the MOSCATO 01 trial. Cancer Discov. 2017;7:586-95.

24. Mullard A. NCI-MATCH trial pushes cancer umbrella trial paradigm. Nat Rev Drug Discov. 2015;14:513-5.

25. Lui VW, Hedberg ML, Li H, Vangara BS, Pendleton K, Zeng $\mathrm{Y}$, et al. Frequent mutation of the PI3K pathway in head and neck cancer defines predictive biomarkers. Cancer Discov. 2013;3:761-9.

26. Tanoue T, Takeichi M. New insights into Fat cadherins. J Cell Sci. 2005;118(Pt 11):2347-53.

27. Morris LG, Kaufman AM, Gong Y, Ramaswami D, Walsh LA, Turcan S, et al. Recurrent somatic mutation of FAT1 in multiple human cancers leads to aberrant Wnt activation. Nat Genet. 2013;45:253-61.

28. Kim KT, Kim BS, Kim JH. Association between FAT1 mutation and overall survival in patients with human papillomavirusnegative head and neck squamous cell carcinoma. Head Neck. 2016;38 Suppl 1:E2021-9.

29. Mandal R, Senbabaoglu Y, Desrichard A, Havel JJ, Dalin MG, Riaz N, et al. The head and neck cancer immune landscape and its immunotherapeutic implications. JCI Insight. 2016;1: e89829.

30. Mehra R, Seiwert TY, Gupta S, Weiss J, Gluck I, Eder JP, et al. 
Efficacy and safety of pembrolizumab in recurrent/metastatic head and neck squamous cell carcinoma: pooled analyses after long-term follow-up in KEYNOTE-012. Br J Cancer. 2018;119: 153-9.

31. Gillison ML, Blumenschein G, Fayette J, Guigay J, Colevas AD, Licitra L, et al. Nivolumab (nivo) vs. investigator's choice (IC) for recurrent or metastatic $(\mathrm{R} / \mathrm{M})$ head and neck squamous cell carcinoma (HNSCC): CheckMate-141. In: Proceedings of the 107th Annual Meeting of the American Association for Cancer Research 2016; 2016 Apr 16-20; New Orleans, LA. Philadelphia, PA: American Association for Cancer Research; 2016. Abstr No. CT099.

32. Gusnanto A, Wood HM, Pawitan Y, Rabbitts P, Berri S. Correcting for cancer genome size and tumour cell content enables better estimation of copy number alterations from next-generation sequence data. Bioinformatics. 2012;28:40-7. 